\title{
Crystallization strategy when no well-diffracted crystals are obtained in the crystallization screening
}

Miki Senda, Toshiya Senda

Structural Biology Research Center, Photon Factory (PF), Institute of Materials Structure Science, High Energy Accelerator Research Organization (KEK)

\begin{abstract}
$\underline{\text { Abstract }}$
In recent years, high-flux beamlines and integrated programs for protein crystallography allow us to determine the crystal structure of proteins with relatively poor crystals. However, high-resolution crystal structures are still required for pharmaceutical science because we need structural details of the target proteins for drug design. Crystallization trial using robots and common screening kits do not always give well-diffracted crystals, therefore, techniques for crystal-quality improvement is crucial to obtain high resolution diffraction data. The process of protein crystallization does not follow routine methods when obtained crystals have insufficient quality. Nonetheless, it is possible to improve the crystal quality by crystal engineering methods; we have so far developed crystal engineering methods on the basis of our experiences such as crystal structure analyses of histone chaperone TAF-Ibeta, CagA oncoprotein from Helicobacter pylori, and GTPsensor PI5P4Kbeta. Here, we present our recent successful examples of crystal quality improvement by anaerobic crystallization and soaking techniques. Our strategy can be applicable to other proteins as well.
\end{abstract}

\section{References}

1. Hayashi et al., (2017) Cell Reports 20, 2876.

2. Senda et al., (2017) Biophys. Reviews, https://doi.org/10.1007/s12551-017-0345-8

3. Senda et al., (2016) Crystal growth \& Design 16, 1565.

4. Sumita et al., (2016) Molecular Cell 61, 1.

5. Hayashi et al., (2012) Cell Host Microbe 12, 20.

6. Muto et al., (2007) PNAS 104, 4285. 